\title{
Design and methodology of SNAP-1: a Sprint National Anaesthesia Project to measure patient reported outcome after anaesthesia
}

Suneetha Ramani Moonesinghe ${ }^{1,2^{*}}$, Eleanor Mary Kate Walker ${ }^{1,2}$, Madeline Bell ${ }^{2}$ and the SNAP-1 investigator group

\begin{abstract}
Background: Patient satisfaction is an important metric of health-care quality. Accidental awareness under general anaesthesia (AAGA) is a serious complication of anaesthesia care which may go unrecognised in the immediate perioperative period but leads to long-term psychological harm for affected patients. The SNAP-1 study aimed to measure patient satisfaction with anaesthesia care and the incidence of AAGA, reported on direct questioning within $24 \mathrm{~h}$ of surgery, in a large multicentre cohort. A secondary aim of SNAP-1 was to test the effectiveness of a new network of Quality Audit and Research Coordinators in NHS anaesthetic departments, to achieve widespread study participation and high patient recruitment rates. This manuscript describes the study methodology.

Methods: SNAP-1 was a prospective observational cohort study. The study protocol was approved by the National Research Ethics Service. All UK NHS hospitals with anaesthetic departments were invited to participate. Adult patients undergoing any type of non-obstetric surgery were recruited in participating hospitals on 13th and 14th May 2014. Demographic data were collected by anaesthetists providing perioperative care. Patients were then approached within $24 \mathrm{~h}$ of surgery to complete two questionnaires - the Bauer patient satisfaction questionnaire (to measure patient reported outcome) and the modified Brice questionnaire (to detect possible accidental awareness). Completion of postoperative questionnaires was taken as evidence of implied consent. Results were recorded on a standard patient case report form, and local investigators entered anonymised data into an electronic database for later analysis by the core research team.

Results: Preliminary analyses indicate that over 15,000 patients were recruited across the UK, making SNAP-1 the largest NIHR portfolio-adopted study in anaesthesia to date. Both descriptive and analytic epidemiological analyses will be used to answer specific questions about the patient perception of anaesthesia care overall and in surgical sub-specialties and to determine the incidence of AAGA.
\end{abstract}

Conclusions: The SNAP-1 study recruited a large number of UK hospitals and thousands of perioperative patients using newly established networks in the UK anaesthetic profession. The results will provide benchmarking information to aid interpretation of patient satisfaction data and also determine the incidence of AAGA reported on a single postoperative visit.

Keywords: Patient-reported outcome, Patient satisfaction, Anesthesia awareness, Epidemiology, Cohort study

\footnotetext{
*Correspondence: rmoonesinghe@gmail.com

${ }^{1}$ UCL/UCLH Surgical Outcomes Research Centre, University College Hospital

NIHR Biomedical Research Centre, London NW1 2BU, UK

${ }^{2}$ National Institute for Academic Anaesthesia's Health Services Research

Centre, Royal College of Anaesthetists, Churchill House 35 Red Lion Square,

London WC1R 4SG, UK
} 


\section{Background}

Patient feedback metrics are viewed as important assessments of the quality of health care. Broadly, the patient's viewpoint can be sought in three domains. First, they can provide feedback on interactions with health-care professionals and specifically communications skills: colloquially, this might be referred to as the "bedside manner" test; and in the UK, this is a mandatory assessment for clinical doctors as part of the revalidation process. Second, patients can provide feedback on their experience of health care; such questionnaires would usually seek the patient viewpoint on a diverse range of issues such as cleanliness of the care environment, efficiency of treatment and services and the courtesy and trustworthiness of staff. Finally, patients may provide their views on the efficacy of their treatment, using patient-reported outcome measures (PROMs). The National Health Service (NHS) has a mandated programme of patient-reported outcome measures for a number of surgical procedures which are aimed at alleviating symptoms and improving health-related quality of life, such as hip and knee joint replacement and varicose vein repairs.

In perioperative anaesthesia practice, the patient perspective on their care can be sought through the administration of patient satisfaction measures. Patient satisfaction has been previously described as a construct which comprises a cognitive evaluation and an emotional response to the care received [1]. It is important to use an appropriately designed questionnaire to measure patient satisfaction, so that reliable and valid results are obtained. A systematic review has recently been published which qualitatively assessed the psychometric development and validation of published patient satisfaction questionnaires relating to anaesthesia practice [2]. This study concluded that the Bauer questionnaire [3] was amongst the best for use in the perioperative setting, both in terms of the rigour with which it was developed and the acceptability of the questionnaire to patients.

While it is of clear importance to measure patient experience and satisfaction, in anaesthetic practice, an important adverse outcome which is not commonly searched for or reported in routine practice is accidental awareness under general anaesthesia (AAGA). Estimates of the incidence of AAGA vary from 1-2 per 1,000 patients in clinical trials, [4-6] to considerably lower (approximately 1 in 15,000 or 20,000 general anaesthetics) in observational studies and surveys [7-9]. As with patient satisfaction, of paramount importance in being able to estimate the incidence of AAGA is the type of questionnaire or interview administered to patients. Traditionally, the Brice questionnaire and protocol has been adopted to elicit reports of AAGA-a series of five open-ended questions which are administered to the patient three times within 30 days of surgery [10]. A modified Brice questionnaire, which used closed questions with multiple choice answers, was subsequently adopted for a large randomised control trial evaluating the efficacy of a protocoled use of $\mathrm{Bi}$-spectral Index monitoring in the prevention of AAGA [6].

While most anaesthetists will be aware of the availability both of patient satisfaction questionnaires and Brice methods, clinical experience tells us that they are infrequently used in routine practice in the United Kingdom (UK). Reasons for this may include a lack of familiarity with validated measures and a lack of benchmarking data to aid interpretation of patient satisfaction data. Furthermore, there are only limited data evaluating the reported incidence of AAGA where patients have been approached using a Brice-style questionnaire in a realworld setting. Therefore, the first UK Sprint National Anaesthesia Project (SNAP-1) aimed to prospectively measure patient reported outcome after anaesthesia in a large multicentre cohort. This paper describes the methods and analysis plan for the SNAP-1 study.

\section{Methods}

\section{Aims and objectives}

The aims of SNAP-1 are to report the descriptive and analytic epidemiology of the delivery and patient-reported outcomes of anaesthesia in the UK. Objectives are as follows:

1. To describe conduct of perioperative anaesthesia in the UK, including personnel, technology support and pharmacological management and postoperative resource utilisation;

2. To describe the characteristics of the patient population undergoing surgery involving an anaesthetist;

3. To measure patient satisfaction after anaesthesia using a validated survey on a national scale;

4. To determine the relationship between individual patient predicted risk using validated scoring systems and patient-reported postoperative outcome

5. To record the reported incidence of AAGA in routine UK practice using a modified Brice questionnaire administered within 24 h of surgery;

6. To determine associations between patient characteristics, procedural factors and adverse patient-reported outcomes

In addition to these main aims and objectives, a secondary aim of SNAP-1 is to determine the effectiveness of the Quality Audit and Research Coordinator (QuARC) network, at achieving local engagement and coordination with a national research project. The QuARC network was established by the National Institute for Academic Anaesthesia's Health Services Research Centre (NIAA HSRC); all UK anaesthetic departments were asked to 
nominate a consultant anaesthetist as the QuARC, with the aim of liaising between the clinical department and the NIAA HSRC in national survey, audit, quality improvement and research projects. A particular aim of SNAP-1 was also to engage trainee anaesthetists in research. Previous surveys have shown that many trainees are unable to access opportunities to participate in research, despite a desire to do so; $[11,12]$ therefore, SNAP1 was proposed as an opportunity to address this gap.

\section{Study design and ethics}

SNAP-1 was a multicentre observational study. Ethics approval was granted by the UK National Research Ethics Service (West Midlands Committee; REC reference 14/WM/0043). The study was approved as a research study, with implied consent being provided by patients upon completion of the questionnaires.

\section{Recruitment, inclusion and exclusion criteria}

All adult ( $\geq 18$ years) patients in participating hospitals undergoing any type of surgery or procedure under anaesthesia (local, regional or general) or sedation administered by an anaesthetist were eligible for inclusion. The following patient groups were excluded:

- Age less than 18 years

- Patients unable to understand spoken or written English

- Obstetric patients

- Patients too unwell or confused to be able to complete the questionnaire

- Patients who refused consent

The recruitment dates for SNAP-1 were 13 and 14 May 2014. All eligible patients having procedures involving an anaesthetist in participating on those dates were given a participant information sheet (Additional file 1) before their procedure, explaining that they would be approached postoperatively to complete the study questionnaires. No specific consent form was required as the ethics approval stated that completion of the follow-up questionnaires could be taken as implied consent: this was explained to patients in the participant information sheet. The target recruitment rate for the study was 7,500 patients.

\section{Dataset}

The dataset comprised three questionnaires: a demographic questionnaire (completed by the perioperative anaesthetist at the time of surgery; Additional file 2), the Bauer patient satisfaction questionnaire and a modified Brice questionnaire (MBQ) (Additional file 3). The MBQ was adapted from that used in the BAG-RECALL study [6]. Demographic data were collected on all patients in participating centres who met inclusion criteria on the study days. The variables within the demographic questionnaire included patient factors, personnel factors and process factors. The patient risk variables were based on simple previously validated preoperative predictors of objective adverse postoperative outcomes. Patients were subsequently approached to complete the follow-up questionnaires (see Additional file 3) either on the day of surgery before hospital discharge (for ambulatory surgery patients) or within $24 \mathrm{~h}$ of surgery (for those undergoing inpatient surgery). Both the date of the procedure and the date of survey completion were noted for each patient. Data were collected on paper case report forms (CRFs) and subsequently transcribed by local investigators into electronic CRFs via a secure web-based data entry portal. A unique identifier for each case was generated through the creation of each new eCRF.

\section{Data management}

The paper CRFs include fields for patient identifiers (name, date of birth and hospital number). These CRFs are securely stored at each participating site in accordance with the principles of information governance and good clinical practice. The electronic CRFs did not contain fields for patient identifiable information: therefore, each local investigator was required to keep a recruitment $\log$ to enable later identification of patients if required. Thus, no patient identifiable data was transferred outside the local hospital environment either electronically or on paper.

\section{Post-study investigator questionnaire}

In order to assess the impact of this study on engagement with research amongst UK anaesthetists, a post-study questionnaire was issued to all registered investigators (see Additional file 4).

\section{Study coordination and funding}

Investigators were sought from every anaesthetic department in the UK which provided perioperative services to adult non-obstetric patients. Site and investigator recruitment was facilitated through the Quality Audit and Research Coordinator (QuARC) network. Recruitment of trainee investigators was particularly encouraged. SNAP-1 was also adopted onto the National Institute for Health Research clinical research portfolio, thereby enabling Clinical Research Network support to be provided to participating hospitals.

Study management was led by a core group of investigators based at the UCL/UCLH Surgical Outcomes Research Centre and the NIAA's HSRC. The core group consisted of the Chief Investigator, a trainee lead investigator and a study administrator based at the NIAA HSRC. Study oversight was provided by the Executive 
Management Board of the NIAA's HSRC. The study sponsor was the University College London Hospitals NHS Foundation Trust. The study was funded through a grant of $£ 18,069$ from the National Institute for Academic Anaesthesia and through salary support (for the Chief Investigator and trainee lead investigator) from the University College London Hospitals NHS Foundation Trust and its National Institute for Health Research Biomedical Research Centre.

\section{Analysis plan}

All results will be reported in accordance with the "Strengthening the Reporting of Observational Studies in Epidemiology" (STROBE) statement [13]. The main focus of analysis is to describe the epidemiology of the practice of anaesthesia and patient-reported outcome after anaesthesia in the overall cohort and, where feasible, in surgical sub-specialty groups and for specific surgical procedures.

\section{Study recruitment and missing data}

Study recruitment rates and missing data will be reported. The total number of sites that participated (broken down by country) will be presented in addition to the total number of patients recruited. The number of eligible patients who did not complete the questionnaires and the reasons reported for this will be stated. Comparison will be made of the demographics of patients who did and did not complete follow-up questionnaires. A description will be provided of any missing data points.

\section{Descriptive epidemiology}

The information collected from the patient demographic and perioperative questionnaire will be presented. The number of cases per specialty and type of procedure will be stated. Point estimates and ranges will be given for patient descriptors (for example, age, ASA grade, comorbidities and body mass index) in addition to detail of the use of long-term analgesics or benzodiazepines. The characteristics of surgery will be described by urgency, severity and length of operation. The personnel (i.e. seniority of the anaesthetist(s)) involved will be described as well as the type and site of anaesthesia induction. Anaesthetic technique and postoperative destination will be described and summarised both overall and for surgical sub-specialty groups.

The variables for three risk prediction/adjustment tools were included in the dataset: the population-based ASA grade; [14] the Surgical Risk Scale; [15] and the Surgical Outcome Risk Tool [16]. The latter two of these systems have been validated for the prediction of 30-day mortality after surgery. The predicted mortality based on these scoring systems will be presented for the overall patient population and for surgical sub-specialty groups. Data will be presented describing patterns of admission to high dependency or intensive care facilities compared with the predicted risk of 30-day mortality according to these systems, in order to assess compliance with national recommendations on postoperative care $[17,18]$.

\section{Bauer patient satisfaction questionnaire}

For each of questions 1-10 (on anaesthesia-related discomfort), counts and percentages will be reported for each of the three potential responses. For questions 1115 (which ask about satisfaction with anaesthesia care), counts and percentages of each of the four potential responses will be reported. Further analysis of the all these responses within different categories using chi-square testing with Bonferroni's correction to compare outcomes between categories, such as (for example):

- Ambulatory surgery-comparisons by specialty and operative procedure

- Inpatient surgery-comparisons by specialty and operative procedure

- Elective/expedited versus immediate/urgent

Within surgical categories, comparison will be made between the responses to the patient satisfaction questionnaires depending on whether the questionnaire was administered on the day of surgery or day 1 postoperatively.

Univariate analysis will be used to demonstrate associations between patient factors and adverse outcomes, defined as the worst category answer for each question. Logistic regression will be used to determine independent risk factors for the adverse outcomes. Analyses will be conducted for the overall population and for surgical and patient categories where the sample size allows. The decision on whether to conduct regression analyses in particular subgroups will be informed by consideration of the principles described by Vittinghoff and McCulloch, including consideration of whether continuous or binary variables are included, the number of events per outcome variables and the sample size itself [19].

\section{Modified Brice questionnaire (MBQ)}

Counts and percentages for the responses to each question will be presented. Comparison will be made between the patients' perception of the anaesthetic technique (general versus sedation or regional) and the actual anaesthetic technique.

The responses to the MBQ will be analysed to identify cases which may potentially represent AAGA. Both the responses to the closed multiple choice questions and free-text responses will be analysed for this purpose. If a case is found where any of these responses would be consistent with a potential case of AAGA, the lead local 
investigator for the site where the patient was anaesthetised will be informed and asked to follow up the case according to their local departmental guidelines. Local investigators will be asked to provide structured feedback to the core research team. Following review of information received from local investigators, the case will be classified as either proven awareness, not awareness, or unable to determine.

As the SNAP-1 study only asks patients about awareness once within $24 \mathrm{~h}$ of surgery, the results will be extrapolated to determine an estimate of the likely actual incidence of awareness, based on the results of previous studies which used three separate interviews (on approximately day 0 , days $1-2$ and day 30 postoperatively) and reported the incidence of awareness occurring on each day [20]. Depending on the event rate and sample size, regression analyses to determine independent risk factors for awareness may be undertaken.

\section{Results and discussion}

SNAP-1 follows similar methodology to previous studies of surgical [21] and anaesthesia-related epidemiology, [22] in adopting a "snapshot" approach to gather a large sample of data in a short time frame. However, these studies did not require patient consent or any interaction with patients outwith the usual practice of the anaesthesia or perioperative team. Thus, to our knowledge, this is the first such study in the UK to measure patientreported outcome related to anaesthesia using this methodology. Preliminary analyses indicate that this is the highest recruiting NIHR portfolio-adopted consenting research study in anaesthesia to date.

SNAP-1 has introduced the concept of using a validated survey to measure patient satisfaction with anaesthesia to the majority of UK hospitals. It is hoped that individual departments will reflect on the resources that were required to facilitate the study and use this to inform future attempts to measure patient satisfaction for the purposes of quality improvement, research and revalidation. The post-study survey will assess the impact of the study on anaesthetists' willingness to engage with the measurement of patient satisfaction and the screening of patients for AAGA in routine practice.

Once analysed and reported, it is hoped that SNAP-1 results will inform the development of patient information leaflets for different types of surgery, which can be used to better inform patients about the likely short-term outcomes of their anaesthetic and surgical experience. Summary national data should provide benchmarking information for future local and national surveys of patient-reported outcome after anaesthesia.

The study has received little primary funding and may provide important data on the feasibility of conducting such large-scale studies on a limited budget. While the study methodology was simple and the intervention (the administration of a questionnaire) was not complex, the logistics of coordinating a study which required individual Research and Development department approval in all four UK-devolved nations was considerable. The post-study investigator questionnaire should improve our understanding of the baseline level of experience of "non-academic" anaesthetists with research and research methodology and the potential benefits (and pitfalls) of engaging with clinical research for both trainees and career-grade doctors. Additionally, it will provide information about the motivation for anaesthetists' participation in this study, including whether the promise of acknowledgment on study manuscripts was a significant incentive to take part. (see Additional file 5).

\section{Conclusions}

SNAP-1 should provide new and important data on patient-reported outcome after anaesthesia, the incidence of AAGA in a "real-world" clinical setting and the impact of participation in nationally coordinated patientfocussed research for clinical anaesthetists in the UK, particularly trainees.

\section{Additional files}

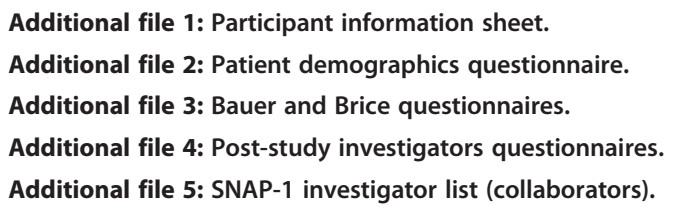

\section{Abbreviations}

AAGA: Accidental awareness under general anaesthesia; SNAP-1: Sprint national anaesthesia project; QuARC: Quality audit and research coordinator; MBQ: Modified brice questionnaire; BPS: Bauer patient satisfaction questionnaire; NIAA: National institute for academic anaesthesia; HSRC: Health services research centre; ASA: American society of anesthesiology physical status score.

\section{Competing interests}

The authors declare that they have no competing interests.

\section{Authors' contributions}

SRM led the study design and conduct and drafted the manuscript. EMKE contributed to the study design and conduct and with SRM drafted the manuscript. MH contributed to the study conduct. All authors approved the final version of the manuscript.

\section{Acknowledgements}

This study was funded by the National Institute for Academic Anaesthesia and the Royal College of Anaesthetists and supported by the National Institute for Health Research University College London Hospitals Biomedical Research Centre.

This manuscript has been written on behalf of the SNAP-1 investigators who contributed to the conduct of the study and are listed in Additional file 5.

Received: 6 October 2014 Accepted: 9 January 2015

Published online: 17 April 2015 


\section{References}

1. Pascoe GC. Patient satisfaction in primary health care: a literature review and analysis. Eval Program Plann. 1983;6:185-210.

2. Barnett SF, Alagar RK, Grocott MP, Giannaris S, Dick JR, Moonesinghe SR Patient-satisfaction measures in anesthesia: qualitative systematic review. Anesthesiology. 2013;119:452-78.

3. Bauer M, Bohrer $\mathrm{H}$, Aichele G, Bach A, Martin E. Measuring patient satisfaction with anaesthesia: perioperative questionnaire versus standardised face-to-face interview. Acta Anaesthesiol Scand. 2001;45:65-72.

4. Myles PS, Leslie K, McNeil J, Forbes A, Chan MT. Bispectral index monitoring to prevent awareness during anaesthesia: the B-Aware randomised controlled trial. Lancet. 2004;363:1757-63.

5. Avidan MS, Zhang L, Burnside BA, Finkel KJ, Searleman AC, Selvidge JA, et al. Anesthesia awareness and the bispectral index. N Engl J Med. 2008:358:1097-108.

6. Avidan MS, Jacobsohn E, Glick D, Burnside BA, Zhang L, Villafranca A, et al. Prevention of intraoperative awareness in a high-risk surgical population. N Engl J Med. 2011;365:591-600.

7. Pandit JJ, Cook TM, Jonker WR, O'Sullivan E. A national survey of anaesthetists (NAP5 baseline) to estimate an annual incidence of accidental awareness during general anaesthesia in the UK. Br J Anaesth. 2013;110:501-9.

8. Pollard RJ, Coyle JP, Gilbert RL, Beck JE. Intraoperative awareness in a regional medical system: a review of 3 years' data. Anesthesiology. 2007;106:269-74

9. Pandit JJ, Andrade J, Bogod DG, Hitchman JM, Jonker WR, Lucas N, et al. 5th National Audit Project (NAP5) on accidental awareness during general anaesthesia: summary of main findings and risk factors. Br J Anaes. 2014. doi:10.1093/bja/aeu313.

10. Brice DD, Hetherington RR, Utting JE. A simple study of awareness and dreaming during anaesthesia. Br J Anaesth. 1970;42:535-42.

11. Ross-Anderson D, Reddy S, Setty S, Moonesinghe SR. Ivory towers and academic utopia: research and audit training in anaesthesia. Bull R Col Anaesth. 2009;58:8-10

12. Mesbah A, Yeung J. Trainees and anaesthesia research: a survey of research activity during UK anaesthesia training. Bull R Coll Anaesth. 2014;85:32-4.

13. von Elm E, Altman DG, Egger M, Pocock SJ, Gotzsche PC, Vandenbroucke JP. The Strengthening the Reporting of Observational Studies in Epidemiology (STROBE) statement: guidelines for reporting observational studies. J Clin Epidemiol. 2008;61:344-9.

14. Saklad M. Grading of patients for surgical procedures. Anesthesiology. 1941;2:281-5

15. Sutton R, Bann S, Brooks M, Sarin S. The surgical risk scale as an improved tool for risk-adjusted analysis in comparative surgical audit. Br J Surg. 2002;89:763-8.

16. Protopapa K, Simpson J, Smith N, Moonesinghe SR. Development and validation of the Surgical Outcome Risk Tool (SORT): a novel preoperative risk stratification tool. Br J Surg. in press.

17. Anderson ID. The higher risk general surgical patient: towards improved care for a forgotton group. Royal College of Surgeons and the Department of: Health; 2011.

18. Findlay GP, Goodwin APL, Protopapa K, Smith NCE, Mason M. Knowing the risk: a review of the perioperative care of surgical patients. National Confidential Enquiry into Patient Outcome and Death. 2011.

19. Vittinghoff $E, M c C u l l o c h ~ C E$. Relaxing the rule of ten events per variable in logistic and Cox regression. Am J Epidemiol. 2007;165:710-8.

20. Sandin $\mathrm{RH}$, Enlund $\mathrm{G}$, Samuelsson $\mathrm{P}$, Lennmarken C. Awareness during anaesthesia: a prospective case study. Lancet. 2000;355:707-11.

21. Pearse RM, Moreno RP, Bauer P, Pelosi P, Metnitz P, Spies C, et al. Mortality after surgery in Europe: a 7 day cohort study. Lancet. 2012;380:1059-65.

22. Sury MRJ, Palmer JHMG, Cook TM, Pandit JJ. The State of UK anaesthesia: a survey of National Health Service activity in 2013. Br J Anaes. 2014; doi:10.1093/bja/aeu292.

\section{Submit your next manuscript to BioMed Central and take full advantage of:}

- Convenient online submission

- Thorough peer review

- No space constraints or color figure charges

- Immediate publication on acceptance

- Inclusion in PubMed, CAS, Scopus and Google Scholar

- Research which is freely available for redistribution

Submit your manuscript at www.biomedcentral.com/submit 